\title{
DOSIMETRY MODELING AND EXPERIMENTAL VALIDATION For the PETALE Program In The CROCUS ReACtor
}

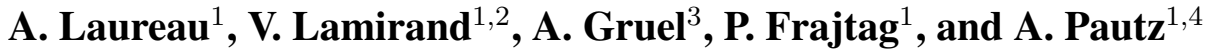 \\ ${ }^{1}$ Laboratory for Reactor Physics and Systems behaviour (LRS) \\ Ecole Polytechnique Fédérale de Lausanne (EPFL), CH-1015 Lausanne, Switzerland \\ ${ }^{2}$ Laboratory for Reactor Physics and Thermal Hydraulics (LRT), Paul Scherrer Institut (PSI) \\ $\mathrm{CH}-5232$ Villigen, Switzerland \\ ${ }^{3}$ CEA/DEN, Experimental Physics, Safety \& Instrumentation Division (SPESI) \\ 13108 St-Paul-Lez-Durance, France \\ ${ }^{4}$ Nuclear Energy and Safety Research Division (NES), Paul Scherrer Institut (PSI) \\ CH-5232 Villigen, Switzerland \\ axel.laureau@epfl.ch
}

\begin{abstract}
The PETALE experimental program in the CROCUS reactor intends to provide integral measurements on reactivity worth and dosimetry measurement to constrain nuclear data relative to stainless steel heavy reflectors. The experimental setup consists in eight successive plates of pure iron, pure nickel, pure chromium, or nuclear-grade stainless steel set at the close periphery of the core. The plates are interleaved with up to nine dosimeters that consist of thin activation foils with different possible materials to be sensitive to different ranges of the neutron spectrum. A precise measurement with a good estimation of the uncertainties and correlations is required, especially when comparing reaction rates, e.g. transmission measurement and/or spectral indices.

The present work focuses on the validation of the dosimetry technics developed in preparation of this experimental program. Different aspects are discussed: monitors, efficiency calibration, self-absorption correction, self-shielding and nuclear data uncertainties. The different sources of uncertainties for the experiment-calculation comparisons are characterized, taking into account all the correlation between the different dosimeters. These correlations are a mandatory element for the aimed Bayesian assimilation in order to avoir overfitting when considering dosimeter providing a similar information.
\end{abstract}

KEYWORDS: Integral experiment, dosimetry, uncertainty propagation, PETALE experimental program

\section{INTRODUCTION}

Dosimetry is an efficient way to measure the neutron flux without introducing an important perturbation thanks to a reduced detection mass and volume. In the PETALE program, various possible 
materials and reactions will allow to be sensitive to different ranges of the neutron spectrum [1,2]. The flux amplitude weighted by the cross section is obtained assuming a very fine characterization of the whole measurement procedure.

We focus in this paper on the validation of the experimental protocol and of the numerical tools developed for the analysis of the transmission phase of the experimental program PETALE. The objective is to compare the Monte Carlo calculations to the test experimental results, taking into consideration all the intermediate aspects such as monitor normalisation, efficiency of the High Purity Germanium (HPGe) spectrometers, dosimeter self-absorption and geometrical corrections. The different sources of uncertainties like the neutron cross sections related to the dosimeter activation reactions will be quantified including their correlation with respect to the different dosimeter positions and materials. This aims at demonstrating the capability to characterize the neutron spectrum with a precision around the percent for the observables of interest in order to be able to provide data for heavy reflector cross section validation or assimilation [3,4]. The experimental description is detailed in section 2 . The experimental results and modeling are respectively presented in sections 3 and 4. Finally, all the detailed intermediary steps are combined in section 5.

\section{EXPERIMENT DESCRIPTION}

\subsection{Experimental setup}

The validation experiment discussed here consists in a series of dosimeters placed at the center of the CROCUS reactor [5]. Different dosimeters have been settled on a plastic plate dedicated to dosimetry measurement at the core center of CROCUS: gold, iron, nickel and aluminium. Each dosimeter has been weighted with a high precision weighing machine, and the position on the plate is visible in figure 1.

The exact thickness cannot be precisely measured because of the very thin dimensions. This quantity is deduced from the volume and the diameter for a better precision. The dosimeter characteristics are summarised in table 1 together with the measured isotope and reaction description.

The plastic plate is positioned above the core during the reactor startup and stabilization. Then the plate falls in the core using a remote system in order to obtain a constant power shape during the irradiation. The positioning of the plate and the dosimeters is displayed in figure 1.

\subsection{Irradiation procedure}

The irradiation time and power are settled to target a statistical uncertainty below $1 \%$ on the counts in the HPGe detector. Thanks to a first rough Monte Carlo neutron transport calculation using the Serpent 2 code [7], the orders of magnitude of the reaction rates in the dosimeters are estimated. Then by associating these reaction rates to the released energy in the core and by assuming a maximum measurement time in the HPGe of around one day, the total energy released has been chosen to $120 \mathrm{Wh}$, or $30 \mathrm{~W}$ during 4 hours for this irradiation.

The power is monitored using a fission chamber during the irradiation and the time series is registered in a text file using the Mirion/Canberra Genie 2000 software. Note that a second monitor is 


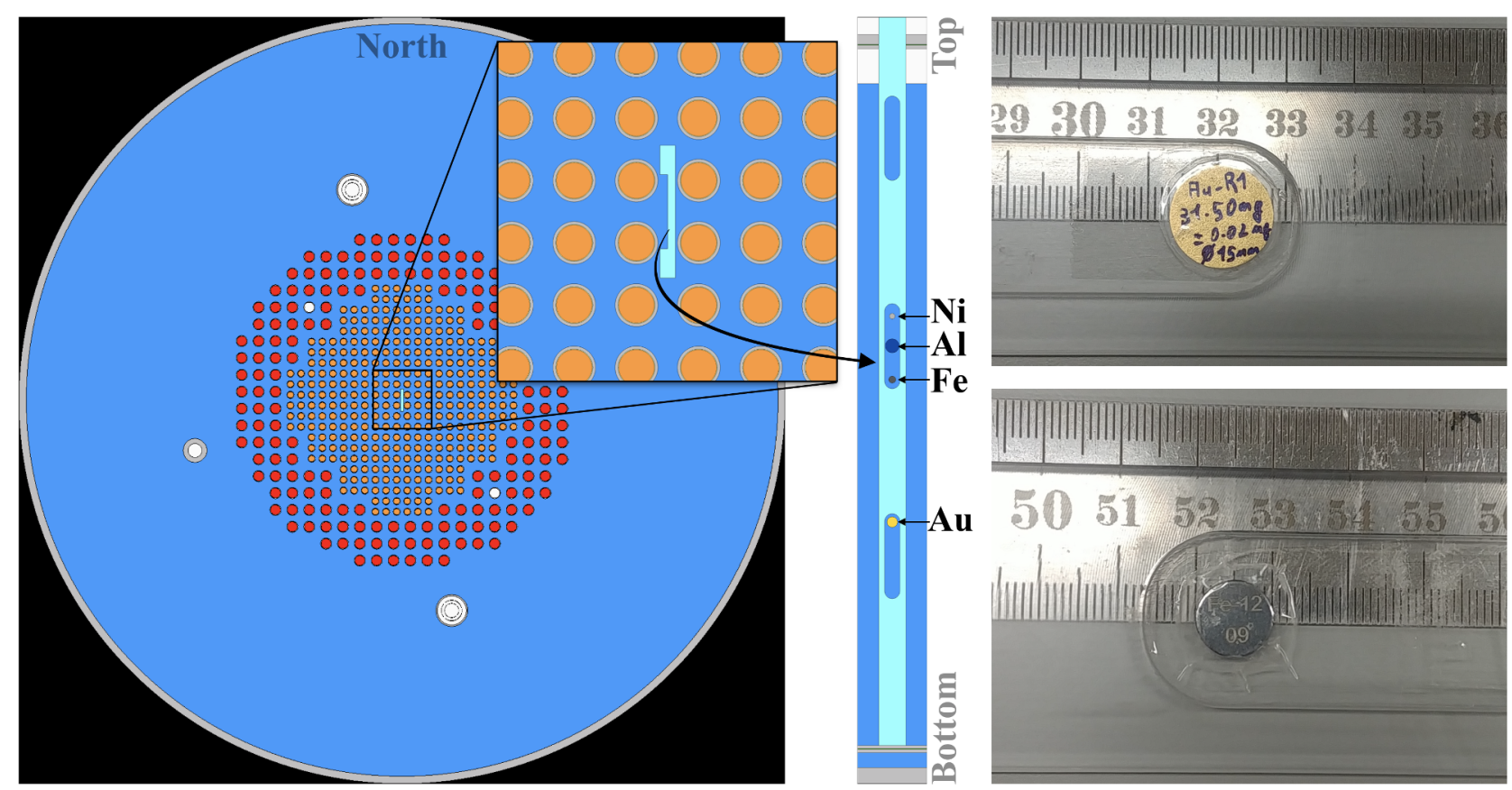

Figure 1: Plastic plate positioning in the CROCUS core using Serpent2 geometry visualization (left) and picture of the dosimeters on the plastic plate (right).

Table 1: Dosimeter description [6]

\begin{tabular}{|c|c|c|c|c|}
\hline Dosimeter & Gold & Iron & Aluminium & Nickel \\
\hline Masse $[\mathrm{mg}]$ & $31.50 \pm 0.02$ & $618.85 \pm 0.02$ & $1734.51 \pm 0.02$ & $834.08 \pm 0.02$ \\
\hline Diameter $[\mathrm{mm}]$ & 15 & 10 & 20 & 8 \\
\hline Position in core $[\mathrm{mm}]$ & $325.5 \pm 0.5$ & $530.0 \pm 0.5$ & $578.5 \pm 0.5$ & $621.0 \pm 0.5$ \\
\hline Reaction & ${ }^{197} \mathrm{Au}(\mathrm{n}, \gamma)^{198} \mathrm{Au}$ & ${ }^{56} \mathrm{Fe}(\mathrm{n}, \mathrm{p})^{56} \mathrm{Mn}$ & ${ }^{27} \mathrm{Al}(\mathrm{n}, \alpha)^{24} \mathrm{Na}$ & ${ }^{58} \mathrm{Ni}(\mathrm{n}, \mathrm{p})^{58} \mathrm{Co}$ \\
\hline Half life & $2.69 \mathrm{~d}$ & $2.58 \mathrm{~h}$ & $15.0 \mathrm{~h}$ & $70.9 \mathrm{~d}$ \\
\hline$\gamma$ - Energy $[\mathrm{keV}]$ & 411.8 & 846.8 & 1368.6 & 810.7 \\
\hline$\gamma$ - Intensity $[\%]$ & $95.62 \pm 0.06$ & $98.85 \pm 0.03$ & $99.9934 \pm 0.0005$ & $99.44 \pm 0.02$ \\
\hline
\end{tabular}

available in CROCUS but dedicated to the operation and not used in the experiment.

After the irradiation, the plastic plate is removed from the core using a rope from the top of the concrete shielding. The dosimeters are moved to the HPGe detection system in the reactor hall in order to measure their activity. 


\section{EXPERIMENTAL RESULTS}

\subsection{Irradiation monitoring}

The monitored power is represented as a function of time in figure 2. Note that the monitor power calibration (counts in the fission chamber per Watt.s) has been determined in a previous work using gold foil dosimeters [8].



Figure 2: Power during the irradiation monitored with a fission chamber.

The dashed line at $t=0 \mathrm{~h}$ represents the start of the irradiation (plastic plate with the dosimeter falling in the core), and the second dashed line at $t=4 \mathrm{~h}$ indicates the reactor stop. This time distribution is the first raw result since it will be used to compute the dosimeter activation. For a reaction measured with a long lifetime such as ${ }^{58} \mathrm{Ni}(\mathrm{n}, \mathrm{p})^{58} \mathrm{Co}$ the final activity is not sensitive to power fluctuations during the experiment. However for reactions with a short lifetime such as ${ }^{56} \mathrm{Fe}(\mathrm{n}, \mathrm{p}){ }^{56} \mathrm{Mn}$, the activated element already starts to decay during the irradiation itself, then the measurement is more sensitive to the end of the irradiation.

\subsection{Measurement of the foil activation}

The second raw result is the number of counts in the corresponding peak in the HPGe given in table 2. The decay of the dosimeters between the irradiation and the measurement is presented in figure 3.

Table 2: Counts in the reaction peak for the different dosimeters

\begin{tabular}{|c|c|c|c|c|}
\hline Dosimeter & Gold & Iron & Aluminium & Nickel \\
\hline Time in HPGe $[\mathrm{h}]$ & 1 & 3 & 22 & 48 \\
\hline Counts & 205464 & 8854 & 37748 & 41514 \\
\hline
\end{tabular}


We observe that, considering the nickel dosimeter, the number of decays is very small before the beginning of the measurement after 150 hours. This cooling is required to ensure that the produced metastable ${ }^{58 m 1}$ Co already decayed ( $\mathrm{T}_{1 / 2}=9$ hours). The limiting factor is the total measurement time in the HPGe, but this is not a problem here since the number of counts is 41514 corresponding to an uncertainty of only $0.5 \%$. On the contrary, considering the iron and aluminium dosimeters the limiting factor is the important decay rate. A longer measurement in the HPGe cannot largely increase the total number of counts. The possible ways to improve the number of counts measured are: increasing the mass of the dosimeters; a larger irradiation time (limited by the saturation as already visible here for iron); and finally a higher power level during the irradiation. Note that, for the gold dosimeter, a cooling time of 6 days allowed to decrease the activity in order to manipulate easily the irradiated dosimeter.

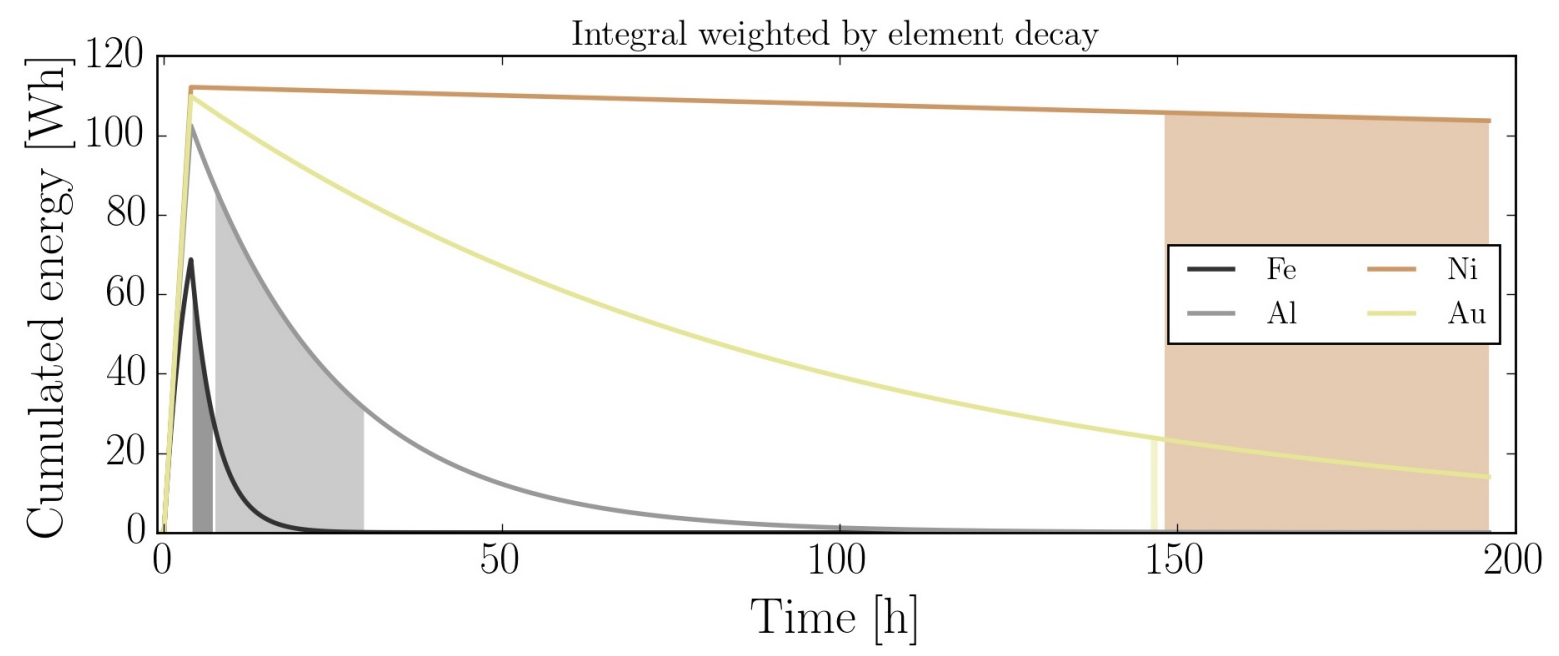

Figure 3: Integral of the power as seen by the dosimeters, with the period in the HPGe represented with the large bands.

The quantity represented in figure 3 is the integral of the power during the irradiation weighted by the decay rate of the different elements, and the large bands represent the time range when the dosimeters are measured in the HPGe. Note that all the equations are summarized in section 5. Multiplying this curve by the reaction rate per Joule provides the number of radioactive nuclei produced, and then the variation of these curves in the HPGe large band represents the amount of decayed nuclei.

The link between the number of decays in the HPGe and the measured count requires: the $\gamma$-ray intensity provided in table 1, the detector efficiency represented in figure 4 , and the equivalence between the source used for the efficiency calibration and the dosimeter (geometrical $+\gamma$ selfabsorption) discussed in the next section. Note that from this efficiency calibration performed with a ${ }^{152} \mathrm{Eu}$ source, the activity uncertainty is around $1 \%$ but this uncertainty might be very correlated between the dosimeters. For example, the iron and nickel $\gamma$-rays are very close (respetively 846 and $810 \mathrm{keV}$ ). Then, as we can see on the correlation matrix, the junction between line $846 \mathrm{keV}$ and column $810 \mathrm{keV}$ provides a correlation close to 1 . For gold $(411 \mathrm{keV})$, the correlation with the other $\gamma$-rays is much smaller, meaning that the uncertainty will not be reduced when comparing two activities. 

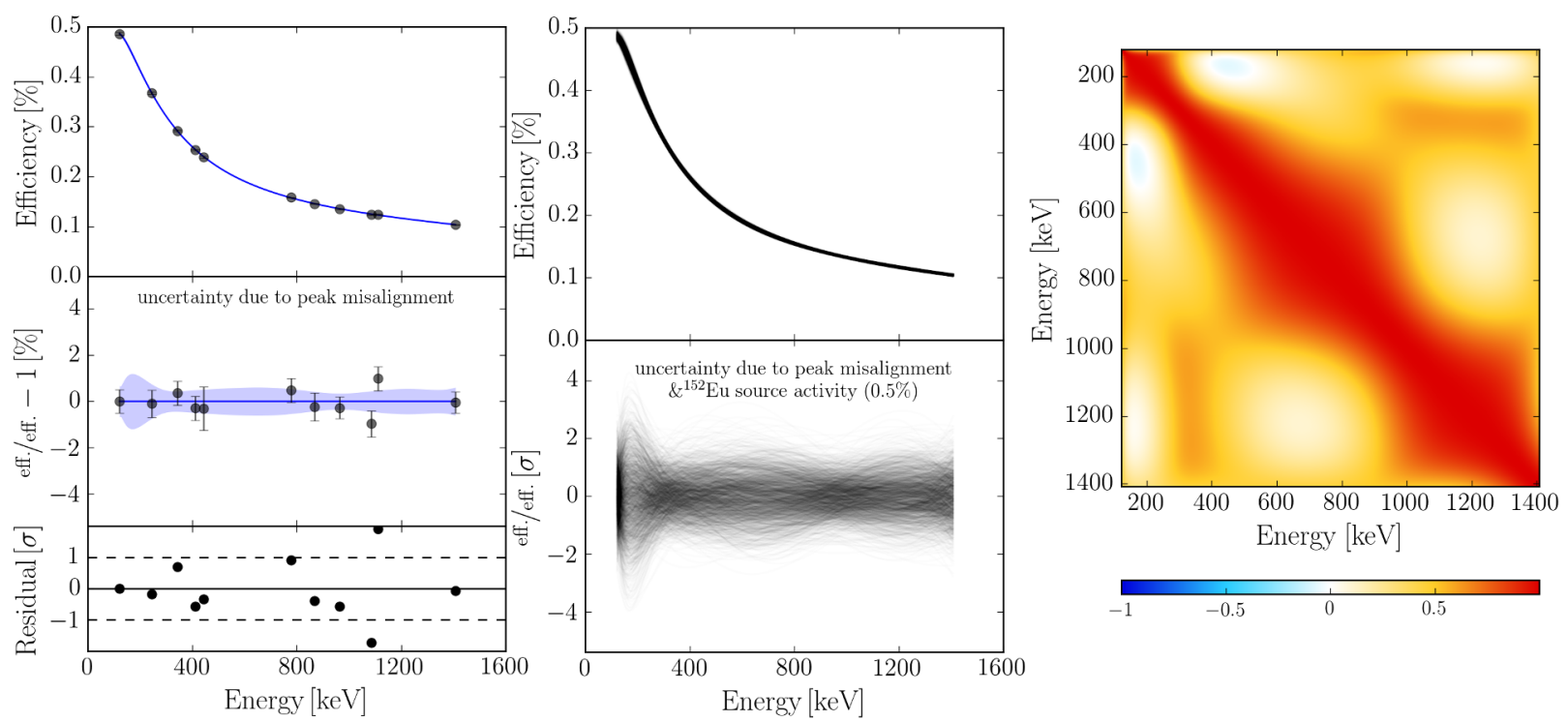

Figure 4: HPGe efficiency calibration procedure using a ${ }^{152}$ Eu source. Left: experimental efficiency as a function of energy (black points) and fitted curve considering only the peak uncertainty (blue curve). Middle: Monte Carlo sampling from correlated fit parameters and incorporation of the $0.5 \%$ source activity uncertainty $(100 \%$ correlated on the spectrum). Right: Covariance matrix associated to the sampled efficiency curves.

\section{MODELING OF THE EXPERIMENT}

\subsection{Modeling of the irradiation}

The whole experiment is extensively modeled using the Serpent 2 code. The reactor representation with the plastic plate and dosimeters inside is represented in figure 1. All the dosimeters are modeled explicitly in order to take into account the neutron self-shielding. The water level is settled to the experimental one.

From this calculation, the reaction rate per source neutron for all the considered reactions is estimated together with the energy released per source neutron for the normalisation. The maximal statistical uncertainty is obtained for the iron dosimeter with $0.65 \%$, lower than the experimental uncertainty.

\subsection{Modeling of the HPGe and the dosimeters}

The HPGe efficiency calibration corresponds to the probability that a gamma emitted in the europium source releases all its energy in the detector.

This efficiency is fully determined by the experimental measurement but an additional correction has to be done on the modeling side. A gamma emitted in a dosimeter is different due to geometrical and self-absorption effects. The europium is not fully representative as a point source for the dosimeters that have a diameter of a few centimeters and a thickness up to a few millimeters. Furthermore a gamma emitted in the dosimeter can be absorbed by the dosimeter itself. For theses 

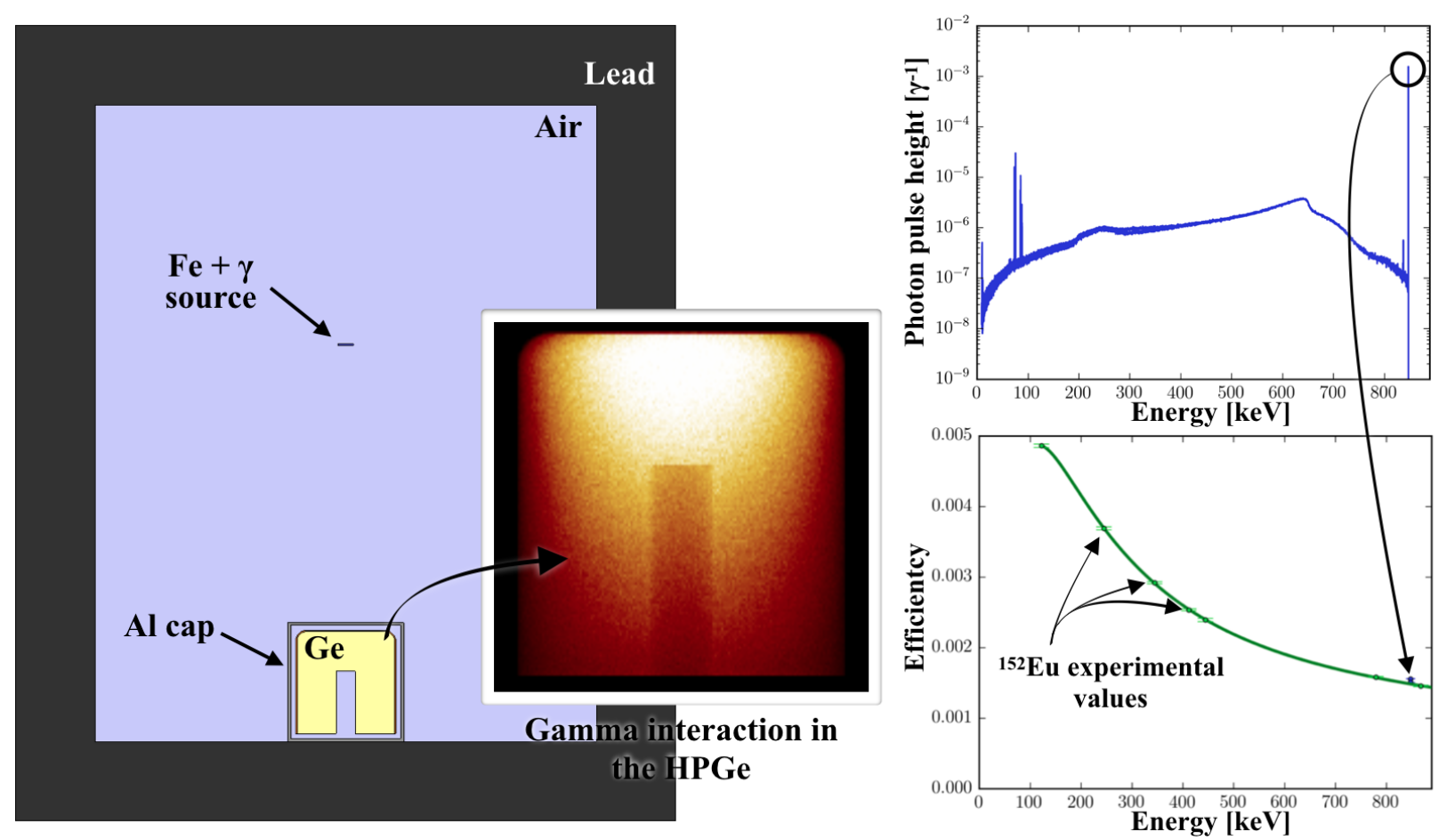

Figure 5: HPGe modelization in Serpent2: geometry and gamma interaction in the cristal (left), photon pulse height detector (top-right), and the corresponding efficiency

(bottom-right) with the experimental values in green for the comparison.

reasons, the HPGe detector is modeled in a Serpent 2 gamma calculation with the europium source and the dosimeter separately. Then the probability that a gamma emitted in both calculations contributes to the photoelectric peak is estimated by running a large amount of gamma emissions, and the ratio between the two gives the geometrical plus self-absorption coefficient. Note that, even if the agreement is very good, the detector does not need to be perfectly modeled since the ratio between the two efficiencies is only sensitive to the europium - dosimeter difference; for example a sensitivity study modifying the radius of the hole in the germanium does not change the correction factor. All the calculations have been done with $10^{9}$ particles. The dosimeter with the largest correction factor obtained is nickel: $93.8 \pm 0.1 \%$ (or $6.2 \pm 0.1 \%$ loss) due to its large thickness and density, meaning that the equivalent HPGe efficiency is lower since some of the gamma rays are lost.

\subsection{Propagation of the nuclear data uncertainty on dosimetry reactions}

The IRDFF dosimetry database [9] has been used for the detector estimation in Serpent2. Together with the estimation of the reaction rate itself, a fine spectrum is computed in order to propagate the dosimetry covariance matrix on the reaction rate using the sandwich rule. In this study the dosimeters are composed of different materials, then there is no correlation between the dosimeters regarding the nuclear data uncertainty on the dosimetry cross section. This would be different if the same dosimeter were used in different positions with a different apparent neutron spectrum. The example of the nickel dosimeter is given in figure 6 . The uncertainty propagation for the iron, 
aluminium, nickel, and gold dosimeters gives respectively $2.7,0.72,1.7$, and $1.1 \%$.
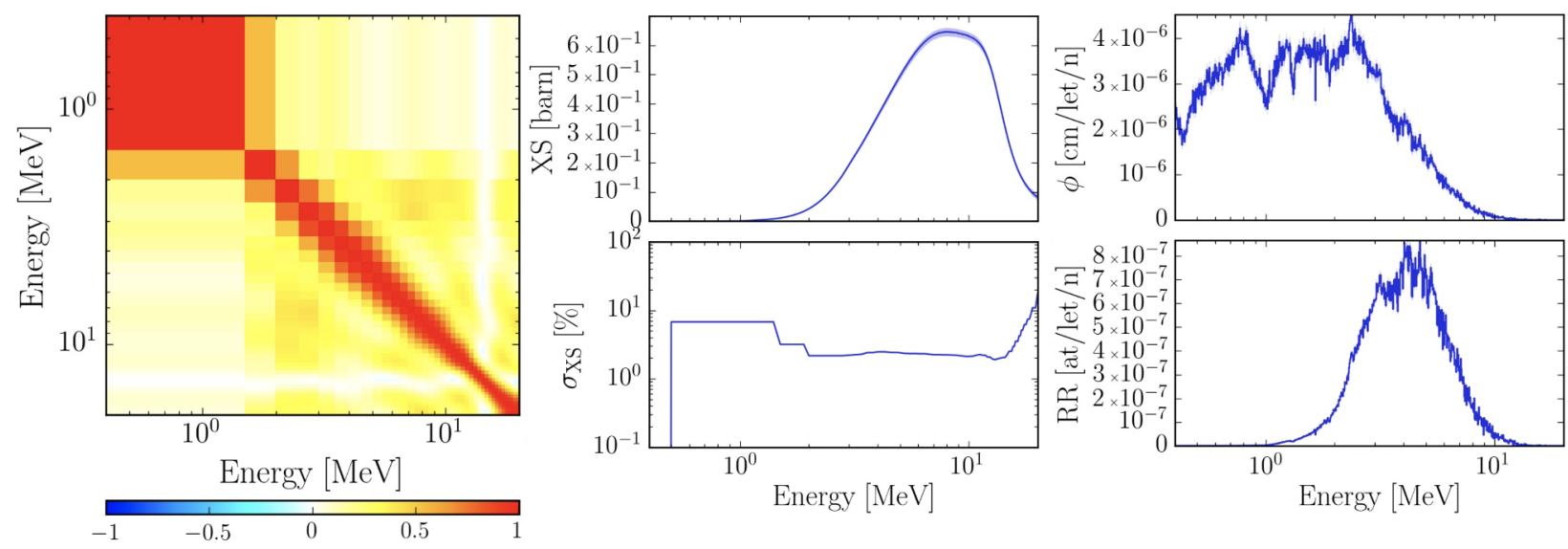

Figure 6: IRDFF covariance (left), cross-section (top-middle) and cross section uncertainties (bottom-middle) used for the uncertainty propagation with the neutron flux (top-right) and reaction rate (bottom-right) spectrum in the nickel dosimeter.

\section{EXPERIMENTAL - CALCULATION COMPARISON}

Finally, the experiment / calculation comparison includes various terms clearly identified as experimental ' $\mathrm{E}$ ' ones, such as the number of counts in the HPGe, and calculation ' $\mathrm{C}$ ' ones such as the reaction rate in the dosimeters estimated by the Monte Carlo code. The raw experimental result is the HPGe measurement, i.e. a number of counts under a peak, and the raw calculated value is a reaction rate per source neutron. The classic comparison element between the ' $C$ ' and the ' $E$ ' values is the dosimeter activity. However this means that the ' $E$ ' activity value is not purely experimental since it includes for example the calculated self-absorption or the modeling of the HPGe efficiency, and these values then contain a covariance. Additionally, the ' $\mathrm{C}$ ' activity value is not purely calculated since it contains the calibration factor of the reactor power (determined by dosimetry), the experimental power shape, and a complex covariance due to the dosimetry uncertainty propagation. In order to avoid this problem, the whole ' $C$ ' to ' $E$ ' values and covariances linking the ' $C$ ' value to the ' $E$ ' value are generated from the different models detailed above. In this way, this factor labeled ' $M$ ' for Model is the only information required, and for example in the frame of a benchmark any contributor only needs to provide ' $\mathrm{C}$ ' reaction rate values. The different terms composing this factor are the following:

- HPGe efficiency: close to the "experiment" but uses models for the fitting procedure. This terms includes uncertainties and covariances.

- Geometrical+self-absorption factor: estimated with Monte Carlo calculations, with an uncertainty and a correlation for all the dosimeters with the same material/geometry/ $\gamma_{\text {ray }}$.

- Gamma emission intensity and decay constant: with uncertainties and correlations.

- Dosimetry cross section uncertainties: propagated on the reaction rate with a Monte Carlo calculation. It contains a strong correlation between the dosimeters if the same reaction is used, but not equal to $100 \%$ if the spectrum is not exactly the same. 
Equation 1 is the ' $C$ ' to ' $\mathrm{E}$ ' factor based on the element discussed in the sections below, starting with the neutron flux $\phi_{i}$ in a dosimeter $i$ normalized per source neutron. The IRDFF dosimetry cross section for dosimeter $i$ is referred to as $\Sigma_{i}$, the decay constant is $\lambda_{i}$, the power in Watt as a function of time is $\mathrm{P}_{\mathrm{W}}(t)$ and the energy released per fission in Joule is $\mathrm{E}_{\mathrm{J}}$. Finally, figure 7 represents ' $C$ ', ' $E$ ' and ' $M$ ' vectors and a correlation matrix for the latter, all the individual components of the correlation matrix being also computed even if only the total is represented due to space constraints.

$$
\begin{aligned}
& \text { Reaction rate per Joule }{ }_{i}=\mathrm{RR}_{i}^{\mathrm{J}}=\frac{1}{\mathrm{E}_{\mathrm{J}}} \int_{\mathrm{E}=0}^{\infty} \phi_{i}(E) \Sigma_{i} \mathrm{dE} \\
& \text { Number of } \operatorname{nuclei}_{i}(t)=\mathrm{N}_{i}^{\text {nuc }}(t)=\int_{t^{\prime}=t_{\text {begin }}^{\text {irrad }}}^{t_{\text {end }}^{\text {irrad }}} \operatorname{RR}_{i}^{J} \cdot P_{\mathrm{W}}\left(t^{\prime}\right) \cdot \exp ^{-\lambda_{i}\left(t-t^{\prime}\right)} \mathrm{d} t^{\prime} \\
& \text { Number of decays in } \mathrm{HPGe}_{i}=\mathrm{N}_{i}^{\text {decay }}=\int_{t=t_{\text {begin }}^{\mathrm{HPGe}}}^{t_{\mathrm{end}}^{\mathrm{HPGe}}} \lambda_{i} \cdot \mathrm{N}_{i}^{\mathrm{nuc}}(t) \mathrm{d} t \\
& \text { Measured counts }=\mathrm{N}_{i}^{\mathrm{HPGe}}=\mathrm{N}_{i}^{\text {decay }}(t) \cdot \epsilon_{i}^{\mathrm{HPGe}} \cdot\left(1-\text { dead time }_{i}\right) \cdot\left(1-\text { auto abs. }_{i}\right)
\end{aligned}
$$

\begin{tabular}{|c|c|c|c|c|c|c|c|c|c|c|c|c|}
\hline Mat & 'C' $\left[\mathrm{at} / \mathrm{n}_{\mathrm{src}}\right]$ & $\sigma_{\mathrm{C}}[\%]$ & ' $M_{C \rightarrow E}$ & $\sigma_{M}[\%]$ & \multicolumn{4}{|c|}{ Correlation [\%] } & 'E' [co & $\sigma_{\mathrm{E}}[\%]$ & $\mathrm{C} / \mathrm{E}-1[\%]$ & $\sigma_{\mathrm{C} / \mathrm{E}}[\%]$ \\
\hline $\mathrm{Fe}$ & 5.82 & 0.65 & & 2.77 & 100 & 7.1 & 8.8 & 0 & & 1.06 & -7.64 & 1 \\
\hline $\mathrm{Al}$ & & & & & 7.1 & & & & & & & 0 \\
\hline $\mathrm{Ni}$ & & 0.22 & & 18 & 8.8 & 10.1 & 100 & 11 & & 0.35 & & 1.82 \\
\hline $\mathrm{Au}$ & $1.2310^{-6}$ & 0.30 & $1.6810^{11}$ & 1.26 & 7.0 & 19.8 & 11.1 & 100 & 205 & 0.2 & 0.5 & 1.32 \\
\hline
\end{tabular}

Figure 7: Calculation (blue) vs experimental (red) comparison using the model (green) with its correlation matrix.

With the experimental results obtained here, the agreement is excellent for the gold dosimeter. One can notice that all the fast dosimeters have a lower count rate of a few percent. This might come from an uncertainty of the fast component modeling such as an uncertainty on the plastic plate radial positioning. It will be investigated in the future using other dosimeter like indium providing both thermal and fast neutron response, and additional Monte Carlo calculation with a displacement of the plastic rod.

\section{CONCLUSIONS}

Different steps are required to link the 'experimental' measured counts in an HPGe to the 'calculated' reaction rate in dosimeter foils estimated by a neutronics Monte Carlo code. These steps are detailed here and the intermediate factors are estimated with their uncertainties. All the uncertainties are provided with their correlations. The latter are very important to reduce uncertainties when comparing two dosimeters, for example with very close gamma ray energies and thus a reduced uncertainty on the HPGe efficiency, or with the same material but in different positions and a similar but not identical neutron spectrum. These components will be important for the analysis of the 
PETALE program. Some factors have been identified for future improvements, such as geometrical uncertainties. They will be studied in parallel of the program whose start is foreseen in early 2020.

\section{REFERENCES}

[1] V. Lamirand, G. Perret, S. Radman, D. Siefman, P. Frajtag, M. Hursin, A. Gruel, P. Leconte, P. Blaise, and A. Pautz. "Design of Separated Element Reflector Experiments in CROCUS: PETALE.” In Reactor Dosimetry: 16th International Symposium. ASTM International (2018).

[2] V. Lamirand, A. Laureau, D. Rochman, G. Perret, A. Gruel, P. Leconte, P. Blaise, and A. Pautz. "An Experimental Programme optimized with Uncertainty Propagation: PETALE in the CROCUS Reactor." EPJ Web of Conferences, volume 211, p. 03003 (2019).

[3] A. Laureau, V. Lamirand, D. Rochman, and A. Pautz. "Total Monte Carlo acceleration for the PETALE experimental programme in the CROCUS reactor." EPJ Web of Conferences, volume 211, p. 03002 (2019).

[4] A. Laureau, V. Lamirand, D. Rochman, and A. Pautz. "Bayesian Monte Carlo assimilation for the PETALE experimental programme using inter-dosimeter correlation." In The International Conference on Nuclear Data for Science and Technology. ND2019, China (2019).

[5] J. Paratte, R. Früh, U. Kasemeyer, M. Kalugin, W. Timm, and R. Chawla. "A benchmark on the calculation of kinetic parameters based on reactivity effect experiments in the CROCUS reactor." Annals of Nuclear Energy, volume 33(8), pp. 739-748 (2006).

[6] M.-M. Bé, V. Chisté, C. Dulieu, M. Kellett, X. Mougeot, A. Arinc, V. Chechev, N. Kuzmenko, T. Kibédi, A. Luca, and A. Nichols. Table of Radionuclides, volume 8 of Monographie BIPM5. Bureau International des Poids et Mesures, Pavillon de Breteuil, F-92310 Sèvres, France (2016). URL http://www.bipm.org/utils/common/pdf/monographieRI/Monographie_BIPM-5 Tables_Vol8.pdf.

[7] J. Leppänen, M. Pusa, T. Viitanen, V. Valtavirta, and T. Kaltiaisenaho. "The Serpent Monte Carlo code: Status, development and applications in 2013." Annals of Nuclear Energy, volume 82, pp. 142-150 (2015).

[8] A. Laureau, V. Lamirand, O. Pakari, P. Frajtag, and A. Pautz. "Power calibration of the CROCUS reactor." submitted to IEEE Trans Nucl Sci (2019).

[9] E. M. Zsolnay, N. Capote, H. J. Nolthenius, A. Trkov, et al. "Summary description of the new international reactor dosimetry and fusion file (IRDFF release 1.0)." Technical report, International Atomic Energy Agency (2012). 